\title{
Preparation of Cross-linked Monodisperse Poly(acrylic acid) Particles by Precipitation Polymerization
}

\author{
Takanori Nakano ${ }^{1}$, Naohiko Saito ${ }^{2 *}$, Hideto Minami ${ }^{*}$ \\ ${ }^{1}$ Department of Chemical Science and Engineering, Graduate School of Engineering, Kobe \\ University, Rokko, Nada, Kobe 657-8501, Japan, E-mail:minamihi@kobe-u.ac.jp \\ ${ }^{2}$ General Center of R\&D, Toagosei Co., LTD., 8 Showacho, Minato-ku, Nagoya, Aichi 455-0026, \\ Japan, E-mail: naohiko_saitou@mail.toagosei.co.jp \\ *Correspondence to Hideto Minami,E-mail:minamihi@kobe-u.ac.jp, Naohiko Saito,E-mail: \\ naohiko_saitou@mail.toagosei.co.jp
}

Number of pages: 4

Number of figures: 3

List of contents

Figure S1. Visual appearances (a, b), and optical micrographs (a', b') of PAA emulsion before (a, a') and after (b, b') addition of acetonitrile/LiBr solution $(2.0 \mathrm{wt} \%)$

Figure S2. Number-averaged size distribution of the obtained PAA particles prepared in ethyl acetate/ethylene carbonate (w/w, (a) 6/4, (b) 5.6/4.4, (c) 5/5, (d) 4.4/5.6)

Figure S3. Molecular weight distribution (with GPC, refractive index (RI) detector, standard: poly(styrene)) of PAA by precipitation polymerization at $500 \mathrm{ppm}$ of water, after methyl esterification 

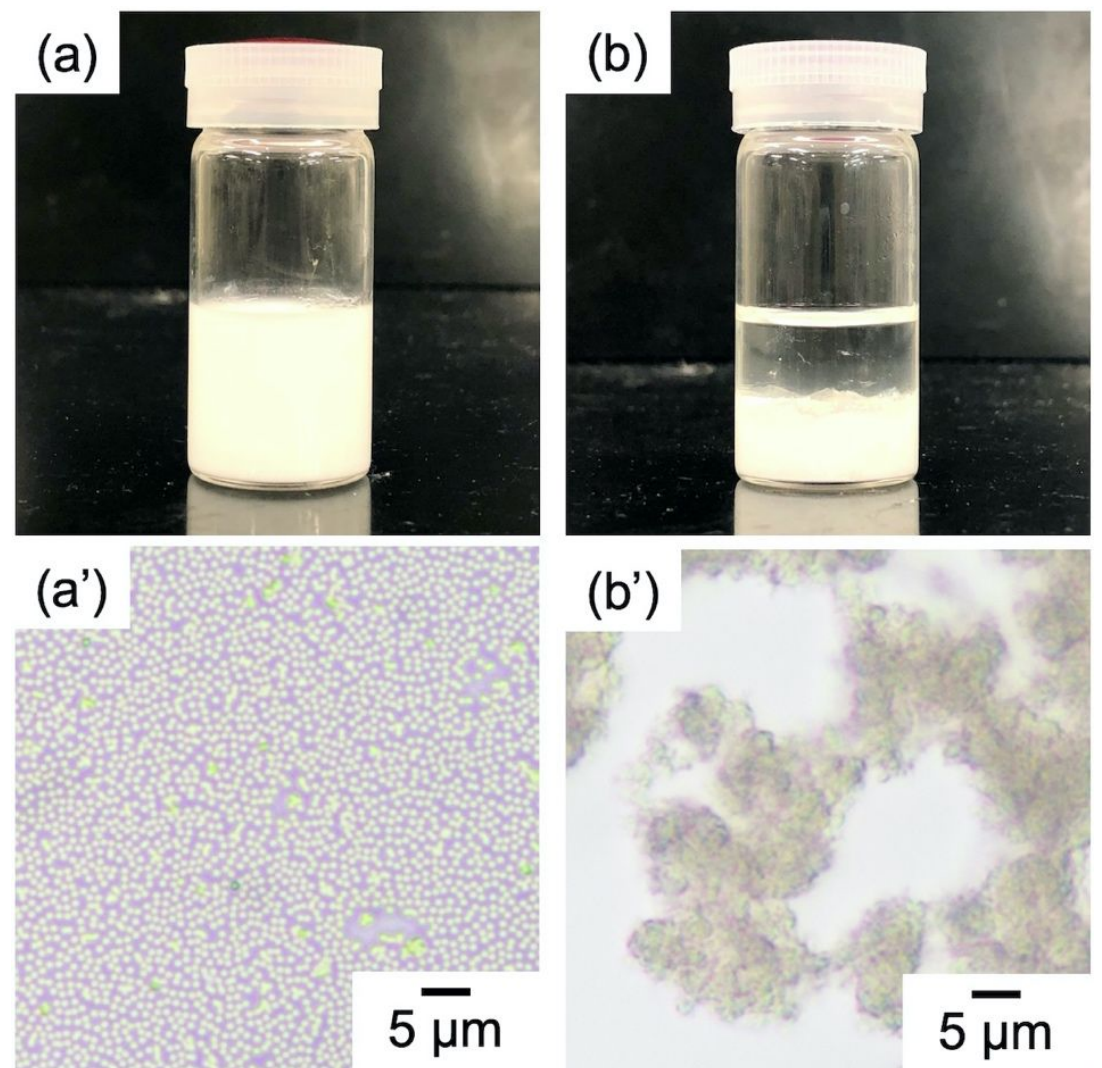

Figure S1. Visual appearances (a, b), and optical micrographs (a', b') of PAA emulsion before (a, a') and after (b, b') addition of acetonitrile/LiBr solution $(2.0 \mathrm{wt} \%)$ 

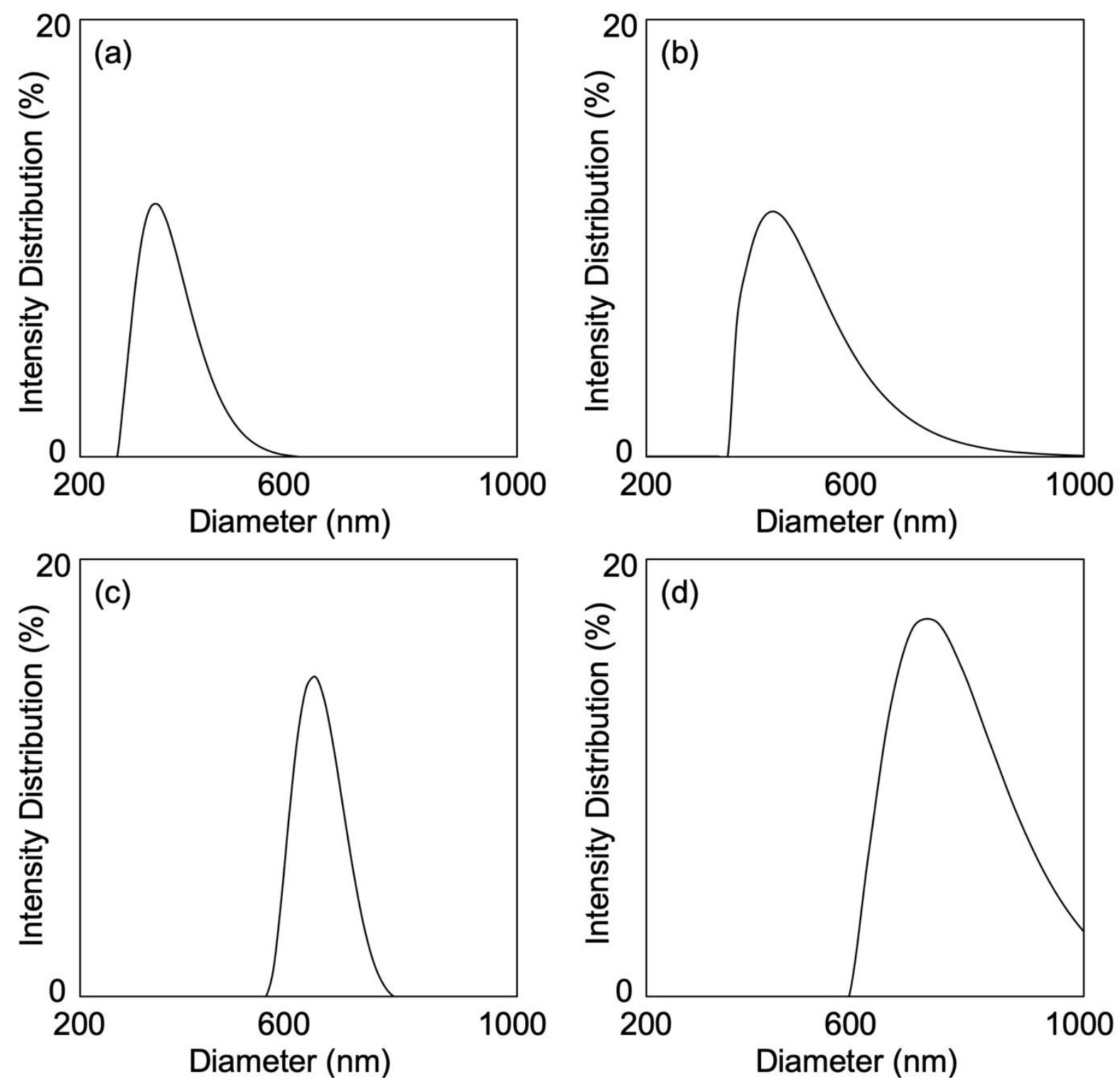

Figure S2. Number-averaged size distribution of the obtained PAA particles prepared in ethyl acetate/ethylene carbonate (w/w, (a) 6/4, (b) 5.6/4.4, (c) 5/5, (d) 4.4/5.6) 


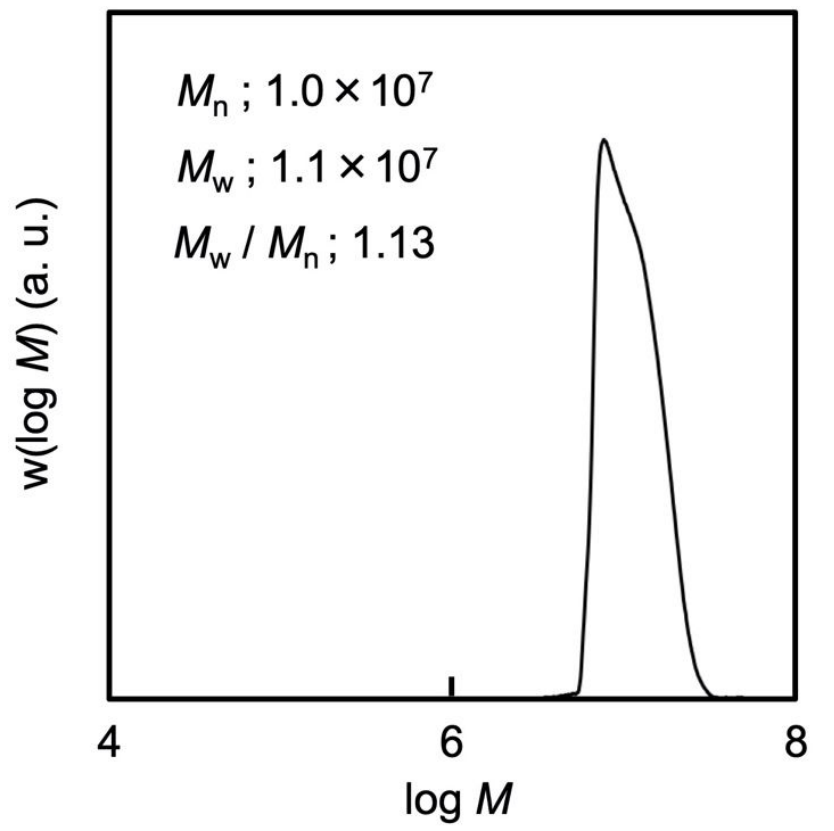

Figure S3. Molecular weight distribution (with GPC, refractive index (RI) detector, standard: polystyrene) of PAA by precipitation polymerization at $500 \mathrm{ppm}$ of water, after methyl esterification 